\title{
Data transfer infrastructure for CMS data taking
}

\author{
Ricky Egeland ${ }^{* a}{ }$ Tony Wildish, ${ }^{b}$ and Simon Metson ${ }^{c}$ \\ ${ }^{a}$ University of Minnesota, Twin Cities \\ ${ }^{b}$ Princeton University \\ ${ }^{c}$ Bristol University \\ E-mail: Ricky.Egeland@cern.ch, Tony.Wildish@cern.ch, \\ Simon. Metsonecern. ch
}

The CMS PhEDEx (Physics Experiment Data Export) project is responsible for facilitating largescale data transfers across the grid ensuring transfer reliability, enforcing data placement policy, and accurately reporting results and performance statistics. The system has evolved considerably since its creation in 2004, and has been used daily by CMS since then. Currently CMS tracks over $2 \mathrm{~PB}$ of data in PhEDEx, and it has been tested well beyond the requirements of CMS. Over the past year PhEDEx has evolved considerably, making use of new technologies (chiefly POE, an asynchronous, event-driven, cooperative-multitasking framework) and to consolidate the various components such that it is easy to reuse existing techniques and components in new features. This has resulted in changes to nearly every piece of the PhEDEx code base, creating a flexible modular framework. We are able to evolve the implementation to match changes in the requirements of the experiment, without changing the fundamental design. Two major new features have recently been added to the PhEDEx system; an extensible data service and an improved transfer backend module. The extensible data service provides machine-readable data over HTTP as the primary means of integration with other CMS services. An authenticated command line interface is also provided, making it possible to provide new utilities quickly with minimal development effort. The new transfer backend module now integrates closely with FTS, the glite provided transfer tool, to provide accurate status information while keeping as much data in flight as possible. The new transfer backend is transfer technology independent, and we expect to be able to support new transfer tools as they become available. We describe the CMS PhEDEx system that is in place for CMS "first data taking" in 2008, provide details on the benefits and implementations of the new features, and describe other new tools that are now available.

XII Advanced Computing and Analysis Techniques in Physics Research November 3-7 2008

Erice, Italy

\footnotetext{
* Speaker.
} 


\section{Introduction}

In order to meet the data distribution requirements $[1,2,3]$ of the CMS [4] experiment at the LHC, the Physics Experiment Data Export (PhEDEx) [5, 6] project was designed to facilitate and manage global data transfers over the grid. Since its conception in 2004, it evolved considerably until version 2.5 was released in early 2007 and has been shown to be a robust, reliable, and scalable system which has guided over 62 million transfers and over 67 PB of data. PhEDEx provides a simple mechanism to request the transfer of thousands of files at a time, which then sets into motion an array of special-purpose software "agents" progressing the transfer state machine to the desired end - files on disk at the destination. Reliability is ensured by independently verifying each file transfer when it lands at the destination, robustness is achieved by intelligently backing off when large numbers of failures are encountered. Through numerous challenges and daily use for months, the transfer management layer of PhEDEx is not in question.

More recent developments have focused on improving the ease of development, the ease of use, and integration of PhEDEx with grid infrastructure such as FTS and SRMv2 as well as with other CMS data management components. These developments did not require extensive redesign of the business logic of core PhEDEx components such as the file router and the site download agents. PhEDEx has adopted the Perl Object Environment (POE) as its scheduling and event manager for the agents. The agents have been modularized into separate libraries for SQL and business logic, for greater re-usability of SQL and simplified debugging. For integration with FTS, a new transfer backend was developed. For integration with other data management components, a web data service for providing XML or JSON via HTTP requests was developed using Apache and mod_perl. Finally, a framework was developed for performing consistency checks to compare database and disk consistency in a controlled fashion. These recent developments are the focus of this paper, following an overview of the project and performance achievements.

\section{Overview of CMS Computing and PhEDEx}

CMS uses the combined resources of over 60 data centers around the world providing over 5 PB of storage space. They are divided into four tiers defined by their service requirements to the experiment. At the host of the experiment, CERN, the Tier 0 is the origin of experiment raw data and the products of first processing. The role of PhEDEx in the Tier 0 is to replicate these irreplaceable data to the various tier 1 centers before the buffers at the Tier 0 become full. CMS must reliably export data at an average rate of $625 \mathrm{MB} / \mathrm{s}$ to the tier 1 centers in order to keep up with data production. The tier 1 centers have a custodial responsibility for safekeeping the Tier 0 data as well as performing processing in order to provide more useful data products for analysis at the tier 2 centers. The tier 2 centers, of which there are over 50, are dedicated to two purposes: to serve as analysis centers for physicists and producing monte carlo data. Analysis products must be transferred to the tier 2 from the tier 1 and tier 2 produced monte carlo data must be transferred to the tier 1 for custodial storage. Finally there are the tier 3 centers which are smaller sites that typically are only interested in receiving data for analysis and have no specific storage or processing responsibilities within CMS. 
PhEDEx manages transfers using a high-availability Oracle database cluster hosted at CERN as a "blackboard" for the system state. This database is known as the Transfer Management Data Base, or TMDB. Software daemon processes or "agents" connect directly to the database to query for the current state and perform the actions necessary to bring the system to the desired state, which is then written back to TMDB. Central agents running at CERN perform most of the intelligence of data routing and transfer task creation. The download agents running at the site receive these tasks and initiate the data transfers using a technology-specific backend interacting with the grid middleware. After a transfer attempt, the file is verified using a site-specific script, and it is the result of that verification which is used to indicate success or failure of the transfer, not any specific return code or message from the grid tools. PhEDEx is a level-triggered as opposed to an edgetriggered system in that it builds large work queues which are largely unaffected by state changes in the steps preceding until the next work cycle. This allows the system to have self-healing properties and avoid widespread damage from isolated errors or transient bugs in a work cycle. The TMDB has been carefully designed to minimize locking contention between the several agents and cache coherency issues by using an row "ownership" model where only one piece of software at a time is expected to act on a given set of rows.

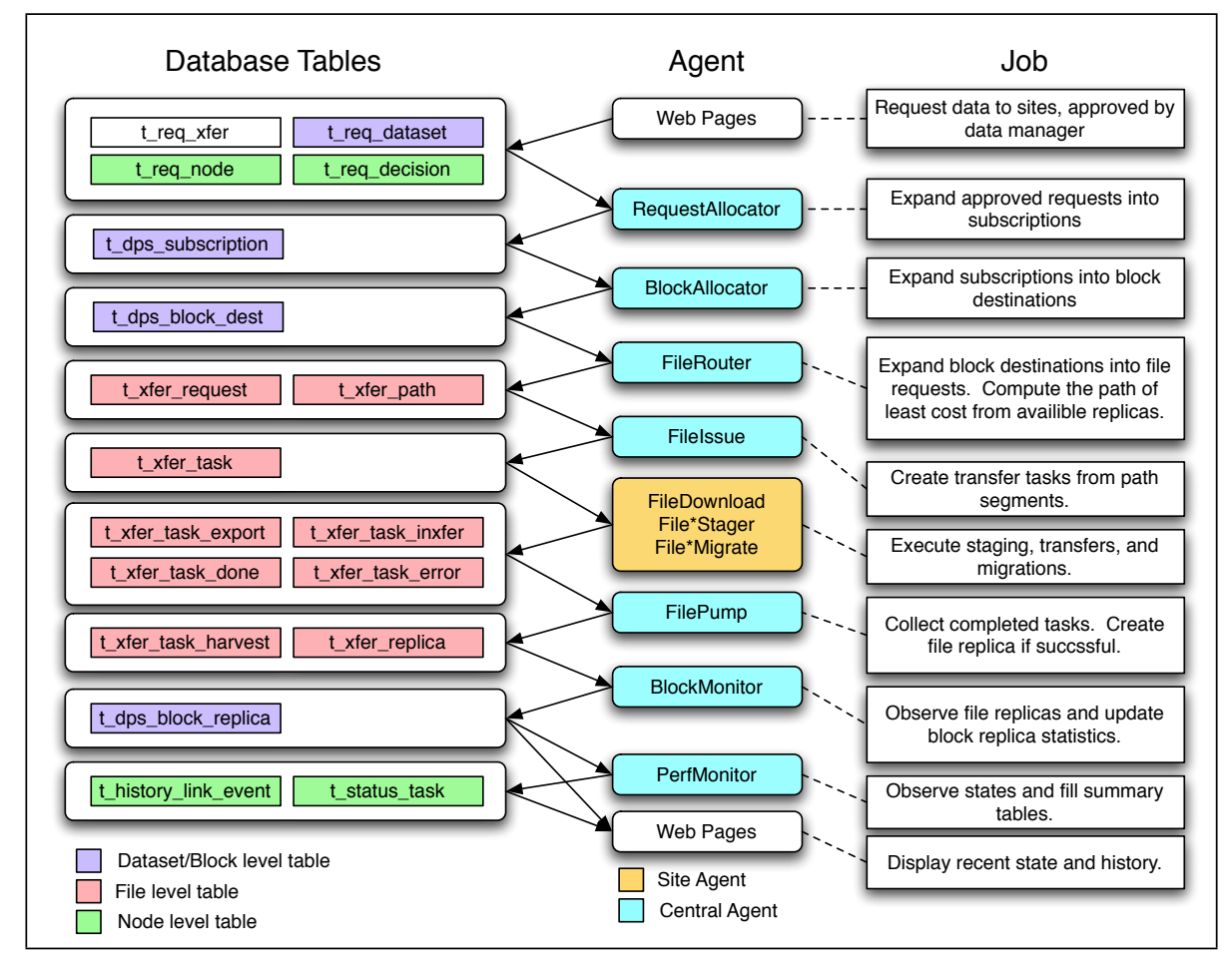

Figure 1: PhEDEx transfer workflow, the relationship between agents and the schema

Users of PhEDEx interact with the system via a web interface which provides administration functions as well as monitoring information. Users are typically CMS operations staff or site administrators, although some physicists interact with the system to subscribe data to their assigned tier 2 center. Users make transfer requests via a simple web form where they indicate the destination nodes and the datasets they wish to transfer. Data managers assigned to each site receive a notification email and make the decision whether to approve or refuse the request. Approved 
requests become subscriptions, which are then handled by the workflow, routing, and finally the site download agents to result in files on the disk at the desired node. Requests for deletion of data at the node are handled in a similar manner, although the workflow is more straightforward as it only involves work for a single node.

Monitoring information is available from the website which represents a recent, but not current state of TMDB. Load on the database is reduced by summarizing monitoring information into special purpose status tables at regular intervals. By building monitoring tools based on these tables instead of those with the current state, response times are reduced and the user experience is improved. Historical information is regularly aggregated from these status tables into variablewidth bins of data on transfer volume, transfer state counts, and number of failures. The monitoring itself introduces little load on the system and the time to access is a function of the time span the user is interested in and is not affected by the current load of the transfer system itself.

\section{Performance and Release Preparation}

$\mathrm{PhEDEx} 3.0$ was extensively tested before release on a dedicated validation cluster in order to ensure no performance issues were introduced by the changes. Two different types of validation runs were performed: a router scaling test which was designed to stress the database and core components to the maximum level possible, and a "data lifecycle" simulation which was designed to exercise all components of the system in a more realistic workflow.

The router scaling test starts from an initial state of 100,000 files evenly distributed across 8 tier 1 and 50 tier 2 nodes, for a total of 66 nodes (tier 1 sites are represented as two nodes, a "Buffer" for outgoing/incoming transfers and the "MSS" (Mass Storage System) which represents tape storage). Each node is then subscribed to all the available data, making the final state 6.6 million replicas. The central agents ran on a dedicated box we had direct access to, so we could monitor the progress of the test. The site agents were run as batch jobs to the computing cluster with each download agent running for up to five nodes simultaneously. No actual file transfers were performed, instead a successful transfer was immediately reported at the point where the transfer would be initiated. The test ran for a total of 10 hours and achieved a replica creation rate of nearly 1 million files per hour for the first 6 hours. The last 4 hours were limited by the test configuration of the tier 1 nodes which prevented them from transferring between them in parallel. If corrected, it is expected that the tier 1 nodes would transfer at the same rate as the tier 2 nodes, and the 4 hour tail would be eliminated. The fictional replication rate achieved in this setup far exceeds the requirements of CMS, and proved that our transfer system will be limited by bandwidth and site storage issues, not the transfer management system..

The lifecycle test is another no-transfer test that exercises more of the PhEDEx code base than the router scaling test and is designed to run for an extended period of time. In this test, the initial state is an empty database, and the "lifecycle agent" simulates the plausible behavior of other CMS components and users. The lifecycle agent regularly injects new file replicas at various nodes at various sizes and frequencies, simulating data production as expected in CMS running. Subscriptions are then made to other nodes and data is transferred. Some of the data is then marked for deletion, simulating that part of the workflow. Furthermore, each of the node-to-node links had a configurable probability for transfer failure, allowing us to exercise error handling and retry 


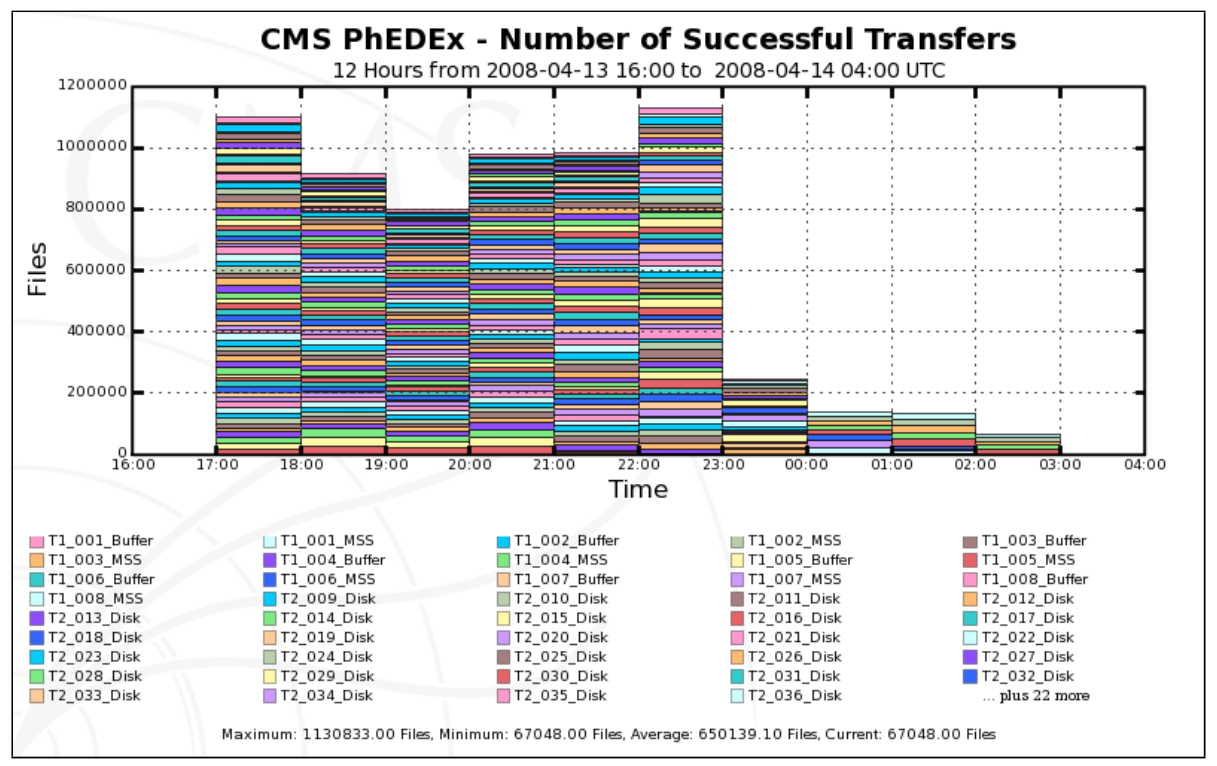

Figure 2: PhEDEx 3.0 scaling test results — number of successful transfers per hour

operations. The entire simulated computing model is dynamically configurable allowing us to test a range of usage patterns. A single scaling factor allowed us to run this simulation at some factor greater than the expected reality. This test ran for two full days and by the end managed 6.5 million files and achieved 14 million replicas. Due to various time constraints we were not able to run this test as cleanly as we would like and fully investigate the results, but the preliminary conclusion was that the system would continue to run well whilst tracking over 2 times more files and replicas than we expected to have in 2009 and at 100x the expected activity rates. The lifecycle agent itself is capable of running even more simulated actions on the system. In future tests we also intend to simulate website and data service usage.

In parallel to the performance validation and scale testing, volunteer site administrators participated in real-world tests of pre-release RPMs of PhEDEx 3.0 using our infinite transfer LoadTest framework. Feedback from these tests gave us fast feedback on oversights and omissions of the release, particularly with respect to the new FTS backend. Fixes were made for the most important concerns, resulting in a release which we were confident could be widely adopted with little disturbance to ongoing activities.

We cannot stress enough the importance of this kind of multi-faceted testing for a major release of distributed computing software. Releasing without absolute confidence in the performance, scalability, and feature set would only result in chaos and many lost man-hours for system recovery.

\section{CCRC'08 Achievements}

PhEDEx 3.0 was released as a mandatory upgrade less than two weeks before the WLCG Combined Computing Readiness Challenge of 2008 (CCRC'08) [7], the most significant computing challenge CMS had participated in up to that point. During CCRC'08, PhEDEx managed the successful transfer of an average of 120 TB per day for 29 days over hundreds of site links, for a 
total of over 3.6 PB of data transferred. $44 \%$ of these transfers were on the production instance, composed of monte-carlo data and with tier 1 tape systems in use. The remaining 56\% was on our "debug" LoadTest instance [8], which creates new transfers from small source samples and does not involve tape systems or permanent data storage.

CCRC'08 was designed to stress the grid systems at a significant fraction of expected start-up levels. CMS set transfer targets for each of the tier 1 sites from the Tier 0 and among its regional tier 2 sites. Nearly all of the transfer targets were met and the transfer infrastructure was considered ready for the expected start up volumes of fall 2008.

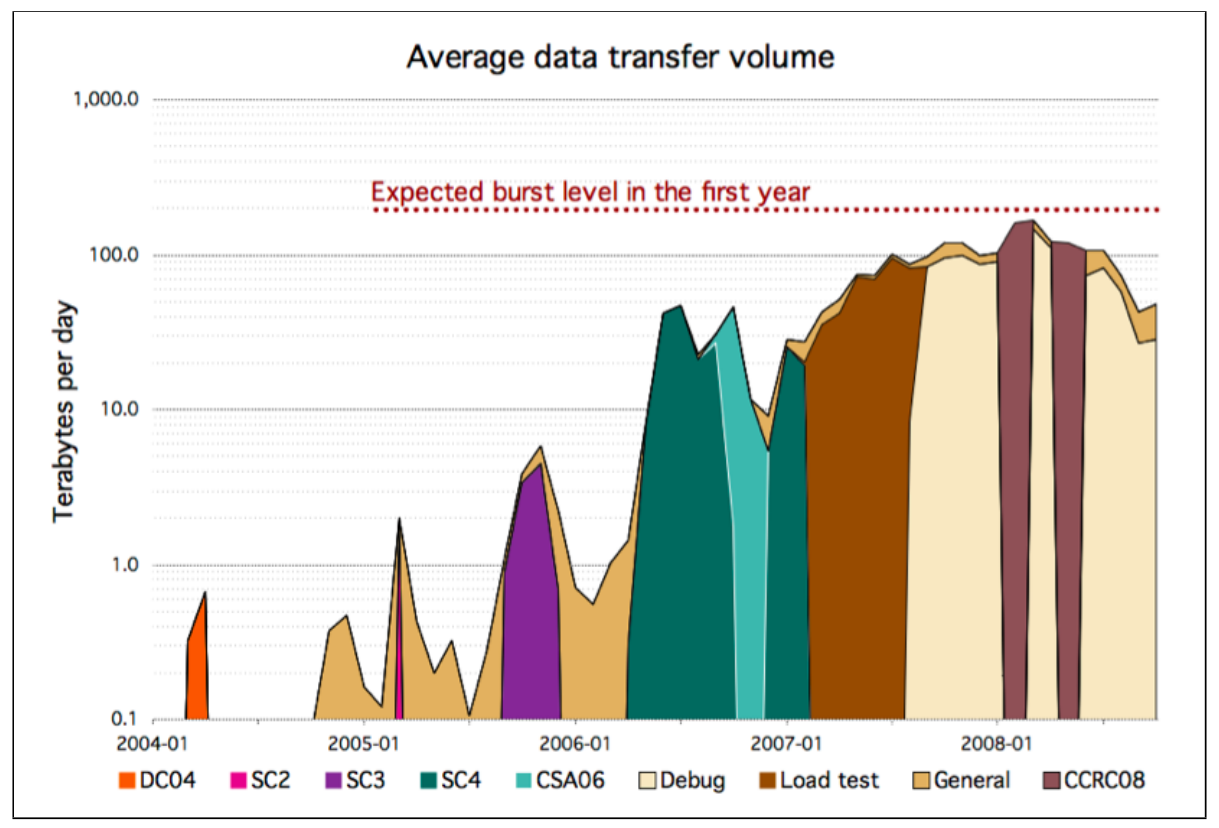

Figure 3: Daily transfer volume using PhEDEx since its creation. Highlighted are the various data challenges, the most recent being CCRC'08/phase 2 which achieved rates of $120 \mathrm{~TB} /$ day.

\section{Data Service}

In order to better integrate with other CMS data and workflow management components, a web data service was developed for PhEDEx. This service is the first public API for exchanging information with the system. The data service provides data in XML, JSON, or perl objects to clients passing API calls and parameters in the request URL. The three data formats are automatically generated from perl objects returned by the web API backend modules. Only authorized users registered in CMS SiteDB with the appropriate role/group for the specific action are allowed. This web service was implemented in Apache/mod_perl and is deployed on a 3 node cluster running in a reverse proxy configuration with a secure front-end for grid certificate authentication.

Currently, the data service provides APIs for data location, file name translations, data injection, and subscribing data. These functions are being integrated with CMS production systems in order to easily automate workflows which require data transfers. Extending the data service is as simple as adding a new perl module to the appropriate namespace, enabling rapid prototyping and 
deployment. A command-line interface is also provided to interact with the data service for both read and write operations. Soon after the release of the data service industrious site administrators in the CMS community began developing custom applications which make use of it for their monitoring. We hope to see this trend continue. Finally, the data service is planned to become the primary data source of a future AJAX-based website.

\section{Use of POE}

POE [9] is the Perl Object Environment, a framework for creating event-driven cooperative multitasking programs in Perl. POE threads, or "sessions", communicate via event-messages with arbitrarily customizable payloads. POE is 10 years old and under active development. It provides several components at all levels of abstraction that make the construction of complex multitasking programs very easy.

Cooperative multitasking in the same process makes the interfaces between components lightweight, easy to develop and debug, and performant. Components can be developed standalone and then plugged into the same process with little effort. For example, a component which monitors the value of some external resource and provides a method to return the result can be developed in isolation and then directly dropped into the program. The traditional problems of race conditions in multi-threaded environments simply do not occur. On the other hand, a single component locking up can block the entire process, so care must be taken to ensure this does not happen. It is intrinsically easier to prevent intra-component lockup than inter-component race conditions, and POE has good support for handling this problem. Database-oriented tasks such as those embodied in PhEDEx are well suited to this type of programming, as transaction-oriented code can be embodied in single event-handlers, running without interruption from other threads.

Use of POE makes it possible to run multiple PhEDEx agents in one process, something that was not possible before. Agents which would in the past have contended for resources on the database can now be managed as cooperative threads, even sharing a database connection. This lowers load and connectivity on the database, and avoids wasted context switches and excessive process load.

\section{The FTS backend}

The core data-movement engines of PhEDEx are the "backend" modules of the FileDownload agent. One of these is the FTS backend, which is responsible for submitting transfer jobs to the glite File Transfer Service (FTS) [10], monitoring those transfers, and acting on their success or failure. A significant feature of PhEDEx 3.0 and its further development through 2008 has been the redesign of the FTS backend to achieve better throughput and reporting with lower load on both the PhEDEx service and the FTS service.

Before PhEDEx 3.0, the FTS backend would prepare a transfer job (of N files to transfer) to submit to the FTS service, submit it, and then wait for the job to complete before examining and acting on the results. A separate subprocess would monitor each transfer job, one subprocess per job, and only when the job ended would that subprocess be harvested by the FTS backend to get the results. A single stuck file would therefore cause knowledge of the state of all the files in the 
job to be delayed, with the consequence that available transfer slots might be left idle unwittingly. Another consequence of this design was that sites often configured their FileDownload agents to transfer only one file per FTS transfer job, in order to avoid the aformentioned idle transfer slots. This meant that an excessive number of transfer jobs were submitted to the FTS service, and that large numbers of transfer monitoring processes could place a heavy load on the machine. Since the transfer monitoring processes were independent, they could also place an unreasonable burden on the FTS service, polling it at a rate which was directly proportional to the number of submitted transfer jobs.

Furthermore, in order to fine-tune bandwidth used per link, sites often configured one FileDownload agent per transfer link, instead of allowing one agent to handle many links. This meant that sites were running many more FileDownload agents than necessary, which translated into many unnecessary connections to the central PhEDEx database. It also meant that the FileDownload agent was deprived of the opportunity to optimize throughput by choosing among links when preparing to submit transfer jobs.

For PhEDEx 3.0 the FTS backend was refactored significantly. FTS transfers are now performed by three interacting components, the remodeled FTS backend, the Queue Monitor, and the Glite interface.

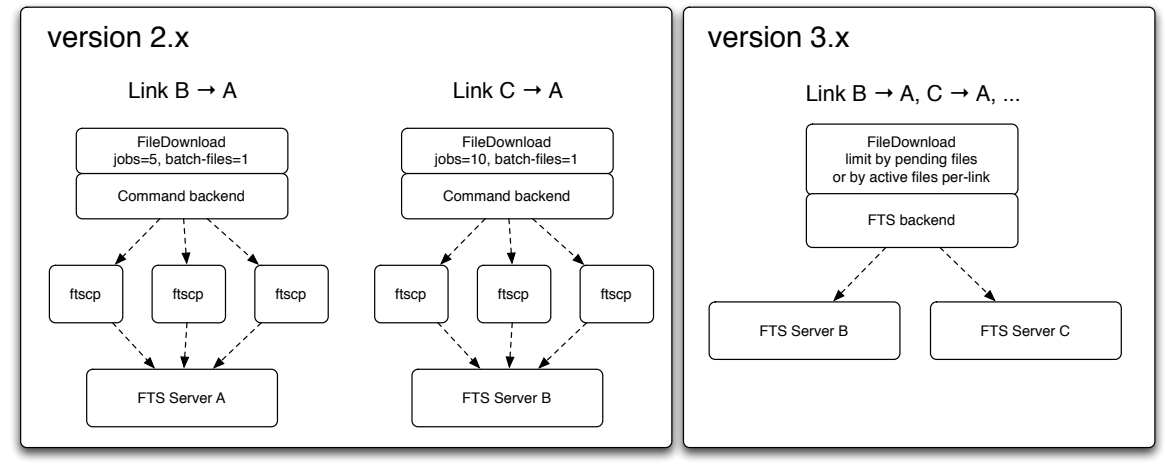

Figure 4: FTS backend in PhEDEx, before and after PhEDEx 3.0

The FTS backend itself takes care of parceling the file queue into discrete chunks that are submitted as FTS transfer jobs, and of harvesting the results and uploading the status to the PhEDEx database. It uses the Glite component to perform the actual submission, decoupling it from the details of the command syntax. The FTS backend also uses the Queue Monitor to determine the state of the jobs that are submitted. It simply notifies the Queue Monitor that a job has been submitted, and lets the Queue Monitor take care of it from then on.

The Queue Monitor maintains a list of transfer jobs that it knows about and periodically checks the status of jobs. It uses the Glite component to execute the monitoring command and return the result. The Queue Monitor checks jobs in a round-robin manner with a fixed interval between checks, so places a constant load on the FTS service regardless of the number of jobs submitted. The POE "postback" mechanism (essentially asynchronous callbacks) allows the monitor to notify the FTS backend of the change of state of each file in a transfer job independently, so the FTS backend can act on completed file transfers (successful or otherwise) while the rest of the files in a transfer job are still in transit. 
The Queue Monitor also maintains details of file state and link state for all the files and transfer links it is monitoring. This information is used by the FTS backend to throttle transfers and to select among links. The information is updated periodically, as each job is monitored, so is not real time, but it does not need to be. The fact that file-state information is available while the transfer job is still executing is enough to make the system adequately performant, as fewer transfer jobs need to be submitted so the interval for monitoring a single job is fairly short.

The Glite component is responsible for constructing the commands for submitting, monitoring, and changing the priority of FTS transfer jobs. It executes the commands and parses the output, returning the result to the calling component as a Perl hash. This encapsulates the interaction with the FTS service completely; nothing else in the system need know any of the details of the command syntax. In PhEDEx 3.0.0 the Glite component executed the commands synchronously, but later releases used POE components (specifically POE::Component::Child) to execute the commands asynchronously, passing the results back using the POE postback mechanism. This was necessary to prevent problems with the glite commands blocking the entire transfer agent.

\section{The Consistency Tools Project}

There are many levels at which inconsistencies can occur between storage systems and internal CMS bookkeeping. Simple database inconsistencies between the transfer service (PhEDEx) and the CMS Dataset Bookkeeping Service (DBS) can arise under some circumstances, files can arrive on disk and then fail to migrate to tape, disks and tapes can fail in many ways, files can become corrupt in copying between disks, and finally, human error cannot be prevented.

With the ever-increasing number of files shipped by PhEDEx, there is a corresponding increase in the effort required to detect and recover from such errors. It is becoming more and more important to have automated tools to check that data really is where we claim it to be. The consistency tools aim to bridge that gap.

The PhEDEx Consistency tools are a set of command-line tools and agents for verifying the consistency of data at a site. They provide a number of lightweight checks for consistency in a controlled manner. "Lightweight" means they provide namespace checks to verify that all files of a block or dataset are in the same state, that files known to PhEDEx are known to DBS and to the underlying Storage Element, that file sizes are consistent with that recorded in PhEDEx, and (where relevant) that migration to tape was successful. By scheduling tests through an agent, instead of running them immediately, we allow the possibility to test large volumes of data without placing undue load on the Storage Element, and without users having to maintain open terminal sessions connected to long-running scripts. Interactive tools are provided, but are not recommended for testing large volumes of data.

The tools do not verify checksums of files, because that would be too resource-intensive. Checksumming all the files at a tier 1 center would be a major undertaking, even if only those on disk were considered. The bandwidth and CPU required to checksum even a modest fraction of the files in a reasonable timescale would be prohibitive. If checksumming were throttled, so that it took several days or weeks to go through all the files available, then the information becomes stale. There is no guarantee that the files that were verified a week ago have not been lost in the meantime. 
In fact this principle pervades the consistency tools design. Any information about consistency between a database and a storage system will have a degree of confidence associated with it, which decays with time. Instead of aiming for the impossible goal of being certain about the state of all data, the tools provide the means to test the state of data at a rate which does not impact the Storage Element, and which can be prioritized to accommodate the most important data first. Rather than attempt to resolve every possible problem, these tools specifically target the needs to reduce the time operators spend in tackling the more frequent problems.

A single BlockConsistency agent is run at each site, configured to have direct local access to the Storage Element. Remote access via SRM is supported, but is too slow to be of much practical use. The agent processes a prioritized queue of requests for a set of PhEDEx nodes that it supports (e.g the Buffer and MSS nodes of a given site) and stores the results back in TMDB. The results are available through the web or by use of a command-line reporting tool. Requests which are deemed too old, because they were not high enough priority to have been processed in time, are expired from the queue.

A single central agent, the BlockDownloadVerifyInjector agent, looks regularly for blocks that are not being transferred in a timely manner. These can be blocks that are delayed in transfer on the WAN or on the LAN (between Buffer and MSS nodes). For blocks that are delayed on the WAN, a test request is scheduled for the source data, checking the filesizes. This should alert operators to transfers which are failing repeatedly because of files that are the wrong size or, more commonly, do not exist at the source for whatever reason. For overdue LAN transfers, tape migration is checked at the destination, to see if there are any problems there.

The BlockDownloadVerifyInjector agent also performs various housecleaning tasks, to prevent excessive growth of the database tables. This helps to ensure that, no matter what volume of data the users request to check, the system will not become overloaded.

The consistency tools were not widely deployed or used until the summer of 2008. Following the first round of feedback, there are ideas for their future development. The most interesting direction is to allow external components to inject tests and examine results programmatically, so that tests can be more readily automated in response to problems detected in other areas of the CMS workflow. For example, a user's analysis job running at a site may encounter errors, which could lead to the automatic verification of the data the user was accessing. Another possibility is to monitor the access to data, by users or other scheduled activity, and to pre-emptively verify the state of associated data, in different data tiers perhaps. The agent-based verification and associated command-line tools provide a sound basis for such further development with little or no risk of impacting more important parts of the system.

\section{Conclusion}

PhEDEx has a long history of transfer management for CMS, and has reliably managed the transfer of petabytes of data. In simulation it has proven its capability for the task well beyond the requirements. In practice it has reliably met the needs of daily grid computing activity and the benchmarks of data challenges. The transfer system is stable over long time scales, and has sustained transfer rates at near the expected burst levels of running at LHC start-up for months. Most of the remaining problems with large scale data transfers fall below the layer which PhEDEx 
occupies, meaning site storage systems and grid transfer tools. Further development of PhEDEx is focused on providing more information for detecting and addressing problems early, as well as improving integration with other components and ease of use. The PhEDEx software platform is ready to meet the challenges of the LHC data-taking era.

\section{References}

[1] CMS Collaboration. The Compact Muon Solenoid computing technical proposal. Technical Report 1996-045, CERN/LHCC, 1996.

[2] CMS Collaboration. The CMS computing project technical design report. Technical Report 2005-023, CERN/LHCC, 2005.

[3] CMS Collaboration. The CMS computing model. Technical Report 2004-035, CERN/LHCC, 2004.

[4] CMS Collaboration. CMS technical proposal. Technical Report 1994-38, CERN/LHCC, 1994.

[5] J. Rehn, T. Barass, D. Bonacorsi, J. Hernandez, I. Semeniouk, L. Tuura, and Y. Wu. PhEDEx high-throughput data transfer management system. In Computing in High Energy Physics 2006 (CHEP06), 2006.

[6] L. Tuura, B. Bockelman, D. Bonacorsi, R. Egeland, D. Feichtinger, S. Metson, and J. Rehn. Scaling CMS data transfer system for LHC start-up. Journal of Physics: Conference Series, 119(7):072030 (10pp), 2008.

[7] L. Buaerdick and D. Bonacorsi. CCRC'08/phase 2: CMS. presentation at CCRC post-mortem workshop - CERN, June 2008.

[8] M.Kadastik, O.Kodolova, J.Letts, and N.Magini. DDT final report: Experience debugging data transfers in CSA07. Internal document, 2008.

[9] Rocco Caputo. POE: The perl object environment, 2003.

http://poe.perl.org/poedown/poe-whitepaper-a4.pdf.

[10] http://egee-jra1-dm.web.cern.ch/egee-jra1-dm/FTS. 